\title{
Singular Positone and Semipositone Boundary Value Problems of Nonlinear Fractional Differential Equations
}

\author{
Chengjun Yuan, ${ }^{1,2}$ Daqing Jiang, ${ }^{1}$ and Xiaojie $\mathbf{X u}^{1}$ \\ ${ }^{1}$ School of Mathematics and Statistics, Northeast Normal University, Changchun 130024, Jilin, China \\ 2 School of Mathematics and Computer, Harbin University, Harbin 150086, Heilongiang, China
}

Correspondence should be addressed to Chengjun Yuan, ycj7102@163.com

Received 8 January 2009; Accepted 15 April 2009

Recommended by Victoria Vampa

We present some new existence results for singular positone and semipositone nonlinear fractional boundary value problem $\mathbf{D}_{0+}^{\alpha} u(t)=\mu a(t) f(t, u(t)), 0<t<1, u(0)=u(1)=u^{\prime}(0)=u^{\prime}(1)=0$, where $\mu>0, a$, and $f$ are continuous, $\alpha \in(3,4]$ is a real number, and $\mathbf{D}_{0+}^{\alpha}$ is Riemann-Liouville fractional derivative. Throughout our nonlinearity may be singular in its dependent variable. Two examples are also given to illustrate the main results.

Copyright (C) 2009 Chengjun Yuan et al. This is an open access article distributed under the Creative Commons Attribution License, which permits unrestricted use, distribution, and reproduction in any medium, provided the original work is properly cited.

\section{Introduction}

Fractional calculus has played a significant role in engineering, science, economy, and other fields. Many papers and books on fractional calculus, and fractional differential equations have appeared recently, (see [1-9]). It should be noted that most of papers and books on fractional calculus, are devoted to the solvability of initial fractional differential equations $($ see $[3,4])$. Here, we consider positive solutions of nonlinear fractional differential equation conjugate boundary value problem involving Riemann-Liouville derivative:

$$
\begin{aligned}
\mathbf{D}_{0+}^{\alpha} u(t) & =\mu a(t) f(t, u(t)), \quad 0<t<1, \\
u(0) & =u(1)=u^{\prime}(0)=u^{\prime}(1)=0,
\end{aligned}
$$

where $\mu>0, a$, and $f$ are continuous. $\alpha \in(3,4]$ is a real number, and $\mathbf{D}_{0+}^{\alpha}$ is the RiemannLiouville fractional derivative. 
It is well known that in mechanics the boundary value problem (1.1) where $\alpha=4$ describes the deflection of an elastic beam rigidly fixed at both ends. The integer order boundary value problem (1.2) has been studied extensively. For details, see for instance, the papers [10-13] and the references therein. In [10, 12], Yao considered

$$
\begin{gathered}
u^{\prime \prime \prime \prime}(t)=\lambda f(t, u(t)), \quad 0<t<1, \\
u(0)=u(1)=u^{\prime}(0)=u^{\prime}(1)=0,
\end{gathered}
$$

and using a Krasnosel'skii fixed-point theorem, derived a $\lambda$-interval such that, for any $\lambda$ lying in this interval, the beam equation has existence and multiplicity on positive solution. In this paper, we will consider a more general situation, namely, the boundary value problem (1.1). To the best of our knowledge, there have been few papers which deal with the boundary value problem (1.1) for nonlinear fractional differential equation.

In this paper, in analogy with boundary value problems for differential equations of integer order, we firstly derive the corresponding Green's function named the fractional Green' function. Consequently problem (1.1) is reduced to an equivalent Fredholm integral equation of the second kind. Finally, using Krasnosel'skii's fixed-point theorems, the existence of positive solutions are obtained.

\section{Preliminaries}

For completeness, in this section, we will demonstrate and study the definitions and some fundamental facts of Riemann-Liouville derivatives of fractional order which can be found in [5].

Definition 2.1 (see [5, Definition 2.1]). The integral

$$
I_{0+}^{\alpha} f(x)=\frac{1}{\Gamma(\alpha)} \int_{0}^{x} \frac{f(t)}{(x-t)^{1-\alpha}} d t, \quad x>0
$$

where $\alpha>0$, is called the Riemann-Liouville fractional integral of order $\alpha$.

Definition 2.2 (see [5, page 36-37]). For a function $f(x)$ given in the interval $[0, \infty)$, the expression

$$
\mathbf{D}_{0+}^{\alpha} f(x)=\frac{1}{\Gamma(n-\alpha)}\left(\frac{d}{d x}\right)^{n} \int_{0}^{x} \frac{f(t)}{(x-t)^{\alpha-n+1}} d t
$$

where $n=[\alpha]+1,[\alpha]$ denotes the integer part of number $\alpha$, is called the Riemann-Liouville fractional derivative of order $\alpha$.

From the definition of Riemann-Liouville derivative, for $\mu>-1$, we have

$$
\mathbf{D}_{0+}^{\alpha} x^{\mu}=\frac{\Gamma(1+\mu)}{\Gamma(1+\mu-\alpha)} x^{\mu-\alpha},
$$

giving in particular $\mathbf{D}_{0+}^{\alpha} x^{\alpha-m}=0, m=1,2,3, \ldots, N$, where $N$ is the smallest integer greater than or equal to $\alpha$. 
Lemma 2.3. Let $\alpha>0$, then the differential equation

$$
\mathrm{D}_{0+}^{\alpha} u(t)=0
$$

has solutions $u(t)=c_{1} t^{\alpha-1}+c_{2} t^{\alpha-2}+\cdots+c_{n} t^{\alpha-n}, c_{i} \in \mathbb{R}, i=1,2 \ldots, n$, as unique solutions, where $n$ is the smallest integer greater than or equal to $\alpha$.

As $\mathbf{D}_{0+}^{\alpha} I_{0+}^{\alpha} u=u$, from Lemma 2.3, we deduce the following statement.

Lemma 2.4. Let $\alpha>0$, then

$$
I_{0+}^{\alpha} \mathbf{D}_{0+}^{\alpha} u(t)=u(t)+c_{1} t^{\alpha-1}+c_{2} t^{\alpha-2}+\cdots+c_{n} t^{\alpha-n},
$$

for some $c_{i} \in \mathbb{R}, i=1,2, \ldots, n, n$ is the smallest integer greater than or equal to $\alpha$.

The following Krasnosel'skii's fixed-point theorem will play a major role in our next analysis.

Theorem 2.5 (see [6]). Let X be a Banach space, and let $P \subset X$ be a cone in X. Assume that $\Omega_{1}, \Omega_{2}$ are open subsets of $X$ with $0 \in \Omega_{1} \subset \bar{\Omega}_{1} \subset \Omega_{2}$, and let $S: P \rightarrow P$ be a completely continuous operator such that, either

(1) $\|S w\| \leq\|w\|, w \in P \cap \partial \Omega_{1},\|S w\| \geq\|w\|, w \in P \cap \partial \Omega_{2}$, or

(2) $\|S w\| \geq\|w\|, w \in P \cap \partial \Omega_{1},\|S w\| \leq\|w\|, w \in P \cap \partial \Omega_{2}$.

Then $S$ has a fixed-point in $P \cap\left(\bar{\Omega}_{2} \backslash \Omega_{1}\right)$.

\section{Green's Function and Its Properties}

In this section, we derive the corresponding Green's function for boundary-value problem (1.1), and obtain some properties of Green's function.

Lemma 3.1. Let $h(t) \in C[0,1]$ be a given function, then the boundary-value problem,

$$
\begin{gathered}
\mathrm{D}_{0+}^{\alpha} u(t)=h(t), \quad 0<t<1,3<\alpha \leq 4, \\
u(0)=u(1)=u^{\prime}(0)=u^{\prime}(1)=0,
\end{gathered}
$$

has a unique solution

$$
u(t)=\int_{0}^{1} G(t, s) h(s) d s
$$

where

$$
G(t, s)=\frac{1}{\Gamma(\alpha)} \begin{cases}t^{\alpha-2}(1-s)^{\alpha-2}[(s-t)+(\alpha-2)(1-t) s]+(t-s)^{\alpha-1}, & 0 \leq s \leq t \leq 1, \\ t^{\alpha-2}(1-s)^{\alpha-2}[(s-t)+(\alpha-2)(1-t) s], & 0 \leq t \leq s \leq 1 .\end{cases}
$$

Here $G(t, s)$ is called Green's function of boundary-value problem (3.1). 
Proof. By means of the Lemma 2.4, we can reduce (3.1) to an equivalent integral equation

$$
u(t)=c_{1} t^{\alpha-1}+c_{2} t^{\alpha-2}+c_{3} t^{\alpha-3}+c_{4} t^{\alpha-4}+\int_{0}^{t} \frac{(t-s)^{\alpha-1}}{\Gamma(\alpha)} h(s) d s .
$$

From $u(0)=u(1)=u^{\prime}(0)=u^{\prime}(1)=0$, we have $c_{3}=c_{4}=0$ and

$$
\begin{aligned}
& c_{1}=\int_{0}^{1} \frac{(1-s)^{\alpha-2}(2 s-\alpha s-1)}{\Gamma(\alpha)} h(s) d s, \\
& c_{2}=\int_{0}^{1} \frac{(\alpha-1)(1-s)^{\alpha-2} s}{\Gamma(\alpha)} h(s) d s .
\end{aligned}
$$

Therefore, the unique solution of (3.1) is

$$
\begin{aligned}
u(t)= & \int_{0}^{1} \frac{t^{\alpha-1}(1-s)^{\alpha-2}(2 s-\alpha s-1)}{\Gamma(\alpha)} h(s) d s+\int_{0}^{1} \frac{t^{\alpha-2}(\alpha-1)(1-s)^{\alpha-2} s}{\Gamma(\alpha)} h(s) d s+\int_{0}^{t} \frac{(t-s)^{\alpha-1}}{\Gamma(\alpha)} h(s) d s \\
= & \frac{1}{\Gamma(\alpha)} \int_{0}^{t}\left[t^{\alpha-1}(1-s)^{\alpha-2}(2 s-\alpha s-1)+t^{\alpha-2}(\alpha-1)(1-s)^{\alpha-2} s+(t-s)^{\alpha-1}\right] h(s) d s \\
& +\int_{t}^{1}\left[t^{\alpha-1}(1-s)^{\alpha-2}(2 s-\alpha s-1)+t^{\alpha-2}(\alpha-1)(1-s)^{\alpha-2} s\right] h(s) d s \\
= & \int_{0}^{1} G(t, s) h(s) d s .
\end{aligned}
$$

The proof is finished.

Lemma 3.2. The function $G(t, s)$ defined by (3.3) has the following properties:

(1) $G(t, s)=G(1-s, 1-t)$, for $t, s \in[0,1]$;

(2) $t^{\alpha-2}(1-t)^{2} q(s) \leq G(t, s) \leq(\alpha-1) q(s)$ and $G(t, s) \leq((\alpha-1)(\alpha-2) / \Gamma(\alpha)) t^{\alpha-2}(1-t)^{2}$ for $t, s \in[0,1]$,

where $q(s)=((\alpha-2) / \Gamma(\alpha)) s^{2}(1-s)^{\alpha-2}$.

Proof. Observing the expression of $G(t, s)$, it is clear that $G(t, s)=G(1-s, 1-t)$ for $t, s \in[0,1]$. In the following, we consider $\Gamma(\alpha) G(t, s)$. 
For $0 \leq s \leq t \leq 1$, we have

$$
\begin{aligned}
\Gamma(\alpha) G(t, s) & =(t-s)^{\alpha-1}-(t-t s)^{\alpha-2}(t-s)+(\alpha-2)(1-t)(t-t s)^{\alpha-2} s \\
& =(t-s)\left[(t-s)^{\alpha-2}-(t-t s)^{\alpha-2}\right]+(\alpha-2)(1-t)(t-t s)^{\alpha-2} s \\
& =-(t-s)(\alpha-2) \int_{t-s}^{t-t s} x^{\alpha-3} d x+(\alpha-2)(t-t s)^{\alpha-2}(1-t) s \\
& \geq-(t-s)(\alpha-2)(t-t s)^{\alpha-3}[(t-t s)-(t-s)]+(\alpha-2)(t-t s)^{\alpha-2}(1-t) s \\
& =-(t-s)(\alpha-2)(t-t s)^{\alpha-3}(1-t) s+(\alpha-2)(t-t s)^{\alpha-2}(1-t) s \\
& =(\alpha-2)(t-t s)^{\alpha-3}(1-t) s[-(t-s)+(t-t s)] \\
& \geq(\alpha-2)(t-t s)^{\alpha-2}(1-t)^{2} s^{2} \\
& =(\alpha-2) t^{\alpha-2}(1-t)^{2} s^{2}(1-s)^{\alpha-2}
\end{aligned}
$$

and

$$
\begin{aligned}
\Gamma(\alpha) G(t, s) & =-(t-s)(\alpha-2) \int_{t-s}^{t-t s} x^{\alpha-3} d x+(\alpha-2)(t-t s)^{\alpha-2}(1-t) s \\
& \leq-(t-s)(\alpha-2)(t-s)^{\alpha-3}[(t-t s)-(t-s)]+(\alpha-2)(t-t s)^{\alpha-2}(1-t) s \\
& =-(t-s)(\alpha-2)(t-s)^{\alpha-3}(1-t) s+(\alpha-2)(t-t s)^{\alpha-2}(1-t) s \\
& =(\alpha-2)(1-t) s\left[(t-t s)^{\alpha-2}-(t-s)^{\alpha-2}\right] \\
& \leq(\alpha-2)^{2}(1-t)^{2} s^{2}(t-t s)^{\alpha-3} \\
& \leq(\alpha-1)(\alpha-2) t^{\alpha-3}(1-t)^{2} s^{2}(1-s)^{\alpha-3} \\
& \leq(\alpha-1)(\alpha-2) s^{2}(1-s)^{\alpha-2} .
\end{aligned}
$$

For $0 \leq t \leq s \leq 1$, since $\alpha>3$, we have

$$
\begin{aligned}
\Gamma(\alpha) G(t, s) & =(t-t s)^{\alpha-2}[(s-t)+(\alpha-2)(1-t) s] \\
& \geq(\alpha-2)(t-t s)^{\alpha-2}(1-t) s \\
& =(\alpha-2) t^{\alpha-2}(1-s)^{\alpha-2}(1-t) s \\
& \geq(\alpha-2) t^{\alpha-2}(1-t)^{2} s^{2}(1-s)^{\alpha-2},
\end{aligned}
$$




$$
\begin{aligned}
\Gamma(\alpha) G(t, s) & =t^{\alpha-2}(1-s)^{\alpha-2}[(s-t)+(\alpha-2)(1-t) s] \\
& \leq t^{\alpha-2}(1-s)^{\alpha-2}[s+(\alpha-2) s] \\
& \leq(\alpha-1) t(1-s)^{\alpha-2} s \\
& \leq(\alpha-1) s^{2}(1-s)^{\alpha-2} \\
& \leq(\alpha-2)(\alpha-1) s^{2}(1-s)^{\alpha-2} .
\end{aligned}
$$

Thus $t^{\alpha-2}(1-t)^{2} q(s) \leq G(t, s) \leq(\alpha-1) q(s)$, for $t, s \in[0,1]$. Combining $G(t, s)=G(1-s, 1-t)$, we have

$$
G(t, s) \leq(\alpha-1) q(1-t)=\frac{(\alpha-1)(\alpha-2)}{\Gamma(\alpha)} t^{\alpha-2}(1-t)^{2}, \text { for } t, s \in[0,1]
$$

This completes the proof.

We note that $u(t)$ is a solution of (1.1) if and only if

$$
u(t)=\mu \int_{0}^{1} G(t, s) a(s) f(u(s)) d s, \quad 0 \leq t \leq 1
$$

For our constructions, we will consider the Banach space $E=C[0,1]$ equipped with standard norm $\|u\|=\max _{0 \leq t \leq 1}\|u(t)\|, u \in X$. We define a cone $K$ by

$$
K=\left\{u \in X \mid u(t) \geq \frac{t^{\alpha-2}(1-t)^{2}}{\alpha-1}\|u\|, t \in[0,1], \alpha \in(3,4]\right\} .
$$

Define an integral operator $A: K \rightarrow X$ by

$$
A u(t)=\mu \int_{0}^{1} G(t, s) a(s) f(u(s)) d s, \quad 0 \leq t \leq 1, u \in K .
$$

Notice from (3.13) and Lemma 3.2 that, for $u \in K, A u(t) \geq 0$ on $[0,1]$ and

$$
A u(t)=\mu \int_{0}^{1} G(t, s) a(s) f(s, u(s)) d s \leq \mu \int_{0}^{1}(\alpha-1) q(s) a(s) f(s, u(s)) d s,
$$

then $\|A u\| \leq \int_{0}^{1}(\alpha-1) q(s) a(s) f(s, u(s)) d s$. 
On the other hand, we have

$$
\begin{aligned}
A u(t) & =\mu \int_{0}^{1} G(t, s) a(s) f(s, u(s)) d s \\
& \geq \mu \int_{0}^{1} t^{\alpha-2}(1-t)^{2} q(s) a(s) f(s, u(s)) d s \\
& \geq \frac{t^{\alpha-2}(1-t)^{2}}{\alpha-1} \mu \int_{0}^{1}(\alpha-1) q(s) a(s) f(s, u(s)) d s \\
& \geq \frac{t^{\alpha-2}(1-t)^{2}}{\alpha-1}\|A u\| .
\end{aligned}
$$

Thus, $A(K) \subset K$. In addition, standard arguments show that $A$ is completely continuous.

\section{Singular Positone Problems}

In this section we present some new result for the singular problem

$$
\begin{aligned}
\mathbf{D}_{0+}^{\alpha} u(t) & =\mu a(t) f(t, u(t)), \quad 0<t<1,3<\alpha \leq 4, \\
u(0) & =u(1)=u^{\prime}(0)=u^{\prime}(1)=0,
\end{aligned}
$$

where $\mu>0$ and nonlinearity $f$ may be singular at $u=0$.

Using Theorem 2.5 we establish the following main result.

Theorem 4.1. Suppose that the following conditions are satisfied.

$$
\begin{aligned}
& a \in C(0,1) \cap L^{1}[0,1] \text { with } a>0 \text { on }(0,1) \\
& f:[0,1] \times(0, \infty) \longrightarrow(0, \infty) \text { is continuous, } \\
& \left\{\begin{array}{l}
f(t, u) \leq g(u)+h(u) \text { on }[0,1] \times(0, \infty) \text { with } g>0 \\
\text { continuous and nonincreasing on }(0, \infty), h \geq 0 \\
\text { continuous on }[0, \infty) \text { and } \frac{h}{g} \text { non decreasing on }(0, \infty),
\end{array}\right. \\
& \exists K_{0} \text { with } g(x y) \leq K_{0} g(x) g(y) \quad \forall x>0, y>0 \text {, } \\
& a_{0}=\mu(\alpha-1) \int_{0}^{1} q(s) a(s) g\left(s^{\alpha-2}(1-s)^{2}\right) d s<\infty, \\
& \exists r>0 \text { with } \frac{r}{g(r)+h(r)}>K_{0}^{2} a_{0} g\left(\frac{1}{\alpha-1}\right),
\end{aligned}
$$




$$
\begin{gathered}
\left\{\begin{array}{l}
\text { There exists } \left.0<\theta<\frac{1}{2} \text { (choose and } f i x \text { it }\right) \text { and a continuous, } \\
\text { nonincreasing function } g_{1}:(0, \infty) \longrightarrow(0, \infty) \text {, and a continuous } \\
\text { function } h_{1}:[0, \infty) \longrightarrow(0, \infty) \text { with } \frac{h_{1}}{g_{1}} \text { nondecreasing on }(0, \infty) \\
\text { and with } f(t, u) \geq g_{1}(u)+h_{1}(u) \text { for }(t, u) \in[\theta, 1-\theta] \times(0, \infty),
\end{array}\right. \\
\left\{\begin{array}{l}
\exists 0<R_{1}<r<R_{2} \text { with }(i=1,2), \\
\frac{R_{i} g_{1}\left(\theta^{\alpha} / \alpha-1\right) R_{i}}{g_{1}\left(R_{i}\right) g_{1}\left(\left(\theta^{\alpha} / \alpha-1\right) R_{i}\right)+g_{1}\left(R_{i}\right) h_{1}\left(\left(\theta^{\alpha} / \alpha-1\right) R_{i}\right)}<\mu \int_{\theta}^{1-\theta} G(\sigma, s) a(s) d s,
\end{array}\right.
\end{gathered}
$$

here $G(t, s)$ is Green's function and

$$
\int_{\theta}^{1-\theta} G(\sigma, s) a(s) d s=\sup _{t \in[0,1]} \int_{\theta}^{1-\theta} G(t, s) a(s) d s .
$$

Then (4.1) has two nonnegative solutions $u_{i}$ with $R_{1}<\left\|u_{1}\right\|<r<\left\|u_{2}\right\|<R_{2}$ and $u_{i}(t)>0$ for $t \in(0,1), i=1,2$.

Proof. First we will show that there exists a solution $u_{2}$ to $(4.1)$ with $u_{2}(t)>0$ for $t \in(0,1)$ and $r<\left\|u_{2}\right\|<R_{2}$. Let

$$
\Omega_{1}=\{u \in E:\|u\|<r\}, \quad \Omega_{2}=\left\{u \in E:\|u\|<R_{2}\right\} .
$$

We now show

$$
\|A u\|<\|u\| \text { for } K \cap \partial \Omega_{1} .
$$

To see this, let $u \in K \cap \partial \Omega_{1}$. Then $\|u\|=\|u\|_{[0,1]}=r$ and $u(t) \geq\left(t^{\alpha-2}(1-t)^{2} /(\alpha-1)\right) r$ for $t \in[0,1]$. So for $t \in[0,1]$, we have

$$
\begin{aligned}
(A u)(t) & =\mu \int_{0}^{1} G(t, s) a(s) f(s, u(s)) d s \\
& \leq \mu \int_{0}^{1}(\alpha-1) q(s) a(s)[g(u(s))+h(u(s))] d s \\
& =\mu \int_{0}^{1}(\alpha-1) q(s) a(s) g(u(s))\left\{1+\frac{h(u(s))}{g(u(s))}\right\} d s
\end{aligned}
$$




$$
\begin{aligned}
& \leq \mu \int_{0}^{1}(\alpha-1) q(s) a(s) g\left(\frac{s^{\alpha-2}(1-s)^{2}}{\alpha-1} r\right)\left\{1+\frac{h(\mathrm{r})}{g(r)}\right\} d s \\
& \leq K_{0} g\left(\frac{r}{\alpha-1}\right)\left\{1+\frac{h(r)}{g(r)}\right\} \mu \int_{0}^{1}(\alpha-1) q(s) a(s) g\left(s^{\alpha-2}(1-s)^{2}\right) d s \\
& =a_{0} K_{0}^{2} g\left(\frac{1}{\alpha-1}\right)[g(r)+h(r)] .
\end{aligned}
$$

This together with (4.7) yields

$$
\|A u\|=\|A u\|_{[0,1]}<r=\|u\|,
$$

so (4.12) is satisfied.

Next we show

$$
\|A u\|>\|u\| \text { for } K \cap \partial \Omega_{2} .
$$

To see this, let $u \in K \cap \partial \Omega_{2}$ so $\|u\|=\|u\|_{[0,1]}=R_{2}$, and let $u(t) \geq\left(t^{\alpha-2}(1-t)^{2} /(\alpha-1)\right) R_{2}$ for $t \in[0,1]$.

We have

$$
\begin{aligned}
(A u)(\sigma) & =\mu \int_{0}^{1} G(\sigma, s) a(s) f(s, u(s)) d s \\
& \geq \mu \int_{\theta}^{1-\theta} G(\sigma, s) a(s)\left[g_{1}(u(s))+h_{1}(u(s))\right] d s \\
& =\mu \int_{\theta}^{1-\theta} G(\sigma, s) a(s) g_{1}(u(s))\left\{1+\frac{h_{1}(u(s))}{g_{1}(u(s))}\right\} d s \\
& \geq g_{1}\left(R_{2}\right) \mu \int_{\theta}^{1-\theta} G(\sigma, s) a(s)\left\{1+\frac{h_{1}\left(\left(s^{\alpha-2}(1-s)^{2} /(\alpha-1)\right) R_{2}\right)}{g_{1}\left(\left(s^{\alpha-2}(1-s)^{2} /(\alpha-1)\right) R_{2}\right)}\right\} d s \\
& \geq g_{1}\left(R_{2}\right) \mu \int_{\theta}^{1-\theta} G(\sigma, s) a(s)\left\{1+\frac{h_{1}\left(\left(\theta^{\alpha} /(\alpha-1)\right) R_{2}\right)}{g_{1}\left(\left(\theta^{\alpha} /(\alpha-1)\right) R_{2}\right)}\right\} d s .
\end{aligned}
$$

This together with (4.9) yields

$$
(A u)(\sigma)>R_{2}=\|u\|
$$

Thus $\|A u\|>\|u\|$, so (4.15) is held.

Now Theorem 2.5 implies that $A$ has a fixed-point $u_{2} \in K \cap\left(\bar{\Omega}_{2} \backslash \Omega_{1}\right)$, that is, $r \leq$ $\left\|u_{2}\right\|=\left\|u_{2}\right\|_{[0,1]} \leq R$ and $u_{2}(t) \geq q(t) r$ for $t \in[0,1]$. It follows from (4.12) and (4.15) that $\left\|u_{2}\right\| \neq r,\left\|u_{2}\right\| \neq R_{2}$, so we have $r<\left\|u_{2}\right\|<R_{2}$. 
Similarly, if we put

$$
\Omega_{1}=\left\{u \in E:\|u\|<R_{1}\right\}, \quad \Omega_{2}=\{u \in E:\|u\|<r\},
$$

we can show that there exists a solution $u_{1}$ to $(4.1)$ with $u_{1}(t)>0$ for $t \in(0,1)$ and $R_{1}<\left\|u_{1}\right\|$ $<r$.

This completes the proof of Theorem 4.1.

Similar to the proof of Theorem 4.1, we have the following result.

Theorem 4.2. Suppose that (4.2)-(4.8) hold. In addition suppose

$$
\left\{\begin{array}{l}
\exists 0<R_{1}<r \text { with } \\
\frac{R_{1} g_{1}\left(\theta^{\alpha} /(\alpha-1)\right) R_{1}}{g_{1}\left(R_{1}\right) g_{1}\left(\theta^{\alpha} /(\alpha-1)\right) R_{1}+g_{1}\left(R_{1}\right) h_{1}\left(\theta^{\alpha} /(\alpha-1)\right) R_{1}}<\mu \int_{\theta}^{1-\theta} G(\sigma, s) q(s) d s .
\end{array}\right.
$$

Then (4.1) has a nonnegative solution $u_{1}$ with $R_{1}<\left\|u_{1}\right\|<r$ and $u_{1}(t)>0$ for $t \in(0,1)$.

Remark 4.3. If in (4.19) we have $R_{1}>r$, then (4.1) a nonnegative solution $u_{2}$ with $r<\left\|u_{2}\right\|<R_{2}$ and $u_{2}(t)>0$ for $t \in(0,1)$.

It is easy to use Theorem 4.2 and Remark 4.3 to write theorems which guarantee the existence of more than two solutions to (4.1). We state one such result.

Theorem 4.4. Suppose that (4.2)-(4.6) and (4.8) hold. Assume that $\exists m \in\{1,2, \ldots\}$ and constants $R_{i}, r_{i}(i=1, \ldots, m)$, with $r_{1}>b_{0}$, and

$$
0<R_{1}<r_{1}<R_{2}<r_{2}<\cdots<R_{m}<r_{m}
$$

In addition suppose for each $i=1, \ldots, m$ that

$$
\frac{r_{i}}{g\left(r_{i}\right)+h\left(r_{i}\right)}>K_{0}^{2} a_{0} g\left(\frac{1}{\alpha-1}\right)
$$

and

$$
\frac{R_{i} g_{1}\left(\theta^{\alpha} /(\alpha-1)\right) R_{i}}{g_{1}\left(R_{i}\right) g_{1}\left(\theta^{\alpha} /(\alpha-1)\right) R_{i}+g_{1}\left(R_{i}\right) h_{1}\left(\theta^{\alpha} /(\alpha-1)\right) R_{i}}<\mu \int_{\theta}^{1-\theta} G(\sigma, s) a(s) d s
$$

hold. Then (4.1) has nonnegative solutions $y_{1}, \ldots, y_{m}$ with $y_{i}(t)>0$ for $t \in(0,1)$.

Example 4.5. Consider the boundary value problem

$$
\begin{array}{r}
\mathbf{D}_{0+}^{\alpha} u(t)=\sigma\left(u^{-a}(t)+u^{b}(t)\right), \quad t \in(0,1), 3<\alpha \leq 4, \\
u(0)=u(1)=u^{\prime}(0)=u^{\prime}(1)=0, \quad 0<a<1<b,
\end{array}
$$


where $\sigma \in\left(0, \sigma_{0}\right)$ is such that

$$
\sigma_{0} \leq \frac{1}{2 a_{1}}
$$

here

$$
a_{1}=\frac{(\alpha-1)(\alpha-2)}{\Gamma(\alpha)} \int_{0}^{1} s^{\alpha-2}(1-s)^{2}\left(s^{\alpha-2}(1-s)^{2}\right)^{-\alpha} d s=\int_{0}^{1} s^{(\alpha-2)(1-a)}(1-s)^{2(1-a)} d s<\infty .
$$

Then (4.23) has two solutions $u_{1}, u_{2}$ with $u_{1}(t)>0, u_{2}(t)>0$ for $t \in(0,1), i=1,2$.

To see this we will apply Theorem 4.1 with (here $0<R_{1}<1<R_{2}$ will be chosen below)

$$
g(u)=g_{1}(u)=u^{-a}, \quad h(u)=h_{1}(u)=u^{b}, \quad a(t)=\sigma, \quad K_{0}=1, \theta=\frac{1}{4} .
$$

Clearly (4.2)-(4.6) and (4.8) hold, and $a_{0}=\left(\sigma a_{1} /(\alpha-1)^{a}\right)$. Now (4.7) holds with $r=1$ since

$$
\frac{r}{g(r)+h(r)}=\frac{1}{2} \geq a_{1} \sigma_{0}>K_{0}^{2} a_{0}(\alpha-1)^{a}=K_{0}^{2} a_{0} g\left(\frac{1}{\alpha-1}\right) .
$$

Finally notice (4.9) is satisfied for $R_{1}$ small and $R_{2}$ large since

$$
\frac{R_{i}}{g_{1}\left(R_{i}\right)\left\{1+\left(h_{1}\right)\left(\left(\theta^{\alpha} / \alpha-1\right) R_{i}\right) /\left(\mathrm{g}_{1}\right)\left(\left(\theta^{\alpha} / \alpha-1\right) R_{i}\right)\right\}}=\frac{R_{i}^{1+a}}{1+(\alpha-1)^{-(a+b)} \theta^{\alpha(a+b)} R_{i}^{a+b}} \longrightarrow 0,
$$

as $R_{1} \rightarrow 0, R_{2} \rightarrow \infty$, since $b>1$. Thus all the conditions of Theorem 4.1 are satisfied so existence is guaranteed.

\section{Singular Semipositone Problems}

In this section we present a new result for the singular semipositone problem:

$$
\begin{aligned}
\mathrm{D}_{0+}^{\alpha} u(t) & =\mu a(t) f(t, u(t)), \quad 0<t<1,3<\alpha \leq 4, \\
u(0) & =u(1)=u^{\prime}(0)=u^{\prime}(1)=0,
\end{aligned}
$$

where $\mu>0$ and nonlinearity $f$ may be singular at $u=0$.

Before we prove our main result, we first state a result.

Lemma 5.1. Suppose $a \in L^{1}[0,1]$ with $a>0$ on $(0,1)$. Then the boundary value problem,

$$
\begin{aligned}
\mathbf{D}_{0+}^{\alpha} u(t) & =a(t) e(t), \quad 0<t<1,3<\alpha \leq 4, \\
u(0) & =u(1)=u^{\prime}(0)=u^{\prime}(1)=0,
\end{aligned}
$$


has a solution w with

$$
w(t) \leq \frac{t^{\alpha-2}(1-t)^{2}}{\alpha-1} C_{0} \quad \text { for } t \in[0,1]
$$

here

$$
C_{0}=\frac{(\alpha-1)^{2}(\alpha-2)}{\Gamma(\alpha)} \int_{0}^{1} a(s) e(s) d s
$$

In fact, from Lemma 3.1, (5.2) has solution

$$
w(t)=\int_{0}^{1} G(t, s) a(s) e(s) d s
$$

According to Lemma 3.2, we have

$$
w(t) \leq \int_{0}^{1} \frac{(\alpha-1)(\alpha-2) t^{\alpha-2}(1-t)^{2}}{\Gamma(\alpha)} a(s) e(s) d s=\frac{t^{\alpha-2}(1-t)^{2}}{\alpha-1} C_{0} .
$$

The above Lemma together with Theorem 2.5 establish our main result.

Theorem 5.2. Suppose that the following conditions are satisfied.

$$
\begin{gathered}
\qquad\left\{\in C(0,1) \cap L^{1}[0,1] \quad \text { with } q>\text { on }(0,1) .\right. \\
\left\{\begin{array}{l}
f:[0,1] \times(0, \infty) \longrightarrow \mathbf{R} \text { is continuous and there exists } \\
\text { a function } e \in C((0,1),(0,+\infty)) \text { with } f(t, u)+e(t) \geq \quad \text { for }(t, u) \in(0,1) \times(0, \infty),
\end{array}\right. \\
\left\{\begin{array}{l}
f^{*}(t, u)=f(t, u)+e(t) \leq g(u)+h(u) \text { on }[0,1] \times(0, \infty) \text { with } g>0 \\
\text { continuous and nonincreasing on }(0, \infty), h \geq 0 \\
\text { continuous on }[0, \infty) \text { and } \frac{h}{g} \text { nondecreasing on }(0, \infty), \\
\quad \exists K_{0} \text { with } g(x y) \leq K_{0} g(x) g(y) \quad \forall x>0, y>0, \\
\quad a_{0}=\int_{0}^{1} G(\sigma, s) q(s) g\left(s^{\alpha-2}(1-s)^{2}\right) d s<\infty, \\
r r C_{0} \text { with } \frac{r}{g\left(\left(r-\mu C_{0}\right) /(\alpha-1)\right)\{1+(h(r) / g(r))\}} \geq \mu K_{0} a_{0},
\end{array}\right.
\end{gathered}
$$




$$
\left\{\begin{array}{l}
\text { There exists } 0<\theta<\frac{1}{2}(\text { choose and fix it) and a continuous, } \\
\text { nonincreasing function } g_{1}:(0, \infty) \longrightarrow(0, \infty), \text { and a continuous } \\
\text { function } h_{1}:[0, \infty) \longrightarrow(0, \infty) \text { with } \frac{h_{1}}{g_{1}} \text { nondecreasing on }(0, \infty) \\
\text { and with } f(t, u)+e(t) \geq g_{1}(u)+h_{1}(u) \text { for }(t, u) \in[\theta, 1-\theta] \times(0, \infty),
\end{array}\right.
$$

and $\exists R>r$ with

$$
\frac{R g_{1}\left(\left(\epsilon \theta^{\alpha} /(\alpha-1)\right) R\right)}{g_{1}(R) g_{1}\left(\left(\epsilon \theta^{\alpha} /(\alpha-1)\right) R\right)+g_{1}(R) h_{1}\left(\left(\epsilon \theta^{\alpha} /(\alpha-1)\right) R\right)} \leq \mu \int_{\theta}^{1-\theta} G(\sigma, s) q(s) d s,
$$

here $\epsilon>0$ is any constant (choose and fix it) so that $1-\left(\mu C_{0} / R\right) \geq \epsilon$ (note $\epsilon$ exists since $R>r>\mu C_{0}$ in fact we can have $\left.\epsilon=1-\left(\mu C_{0} / r\right)\right)$ and $G(t, s)$ is Green's function and

$$
\int_{\theta}^{1-\theta} G(\sigma, s) a(s) d s=\sup _{t \in[0,1]} \int_{\theta}^{1-\theta} G(t, s) a(s) d s .
$$

Then (5.1) has a solution $y \in C[0,1] \cap C^{2}(0,1)$ with $y(t)>0$ for $t \in(0,1)$.

Proof. To show that (5.1) has a nonnegative solution we will look at the boundary value problem

$$
\begin{aligned}
\mathbf{D}_{0+}^{\alpha} y(t) & =\mu q(t) f^{*}(t, y(t)-\phi(t)), \quad 0<t<1,3<\alpha \leq 4, \\
y(0) & =y(1)=y^{\prime}(0)=y^{\prime}(1)=0,
\end{aligned}
$$

where

$$
\phi(t)=\mu M w(t), \quad 0 \leq t \leq 1,
$$

( $w$ is as in Lemma 5.1).

We will show, using Theorem 2.5 , that there exists a solution $y_{1}$ to (5.16) with $y_{1}(t)>$ $\phi(t)$ for $t \in(0,1)$. If this is true then $u(t)=y_{1}(t)-\phi(t), 0 \leq t \leq 1$ is a nonnegative solution (positive on $(0,1))$ of $(5.1)$, since

$$
\begin{aligned}
\mathbf{D}_{0+}^{\alpha} u(t)=\mathbf{D}_{0+}^{\alpha} y_{1}(t)-\mathbf{D}_{0+}^{\alpha} \phi(t) & =-\mu q(t) f^{*}\left(t, y_{1}(t)-\phi(t)\right)+\mu q(t) e(t) \\
& =-\mu q(t)\left[f\left(t, y_{1}(t)-\phi(t)\right)+e(t)\right]+\mu q(t) e(t) \\
& =-\mu q(t) f\left(t, y_{1}(t)-\phi(t)\right) \\
& =-\mu q(t) f(t, u(t)), \quad 0<t<1 .
\end{aligned}
$$

As a result, we will concentrate our study on (5.16). Let $E, K$ as in Section 2, and let

$$
\Omega_{1}=\{u \in E:\|u\|<r\}, \quad \Omega_{2}=\{u \in E:\|u\|<R\} .
$$


Next let $A: K \cap\left(\bar{\Omega}_{2} \backslash \Omega_{1}\right) \rightarrow E$ be defined by

$$
(A y)(t)=\mu \int_{0}^{1} G(t, s) q(s) f^{*}(s, y(s)-\phi(s)) d s, \quad 0 \leq t \leq 1 .
$$

In addition, standard argument shows that $A(P) \subset P$ and $A$ is completely continuous.

We now show

$$
\|A y\| \leq\|y\| \text { for } K \cap \partial \Omega_{1} .
$$

To see this, let $y \in K \cap \partial \Omega_{1}$. Then $\|y\|=\|y\|_{[0,1]}=r$ and $y(t) \geq\left(t^{\alpha-2}(1-t)^{2} /(\alpha-1)\right) r$ for $t \in[0,1]$. Now for $t \in(0,1)$, the Lemma 5.1 implies

$$
y(t)-\phi(t) \geq \frac{t^{\alpha-2}(1-t)^{2}}{\alpha-1} r-\mu \frac{t^{\alpha-2}(1-t)^{2}}{\alpha-1} C_{0} \geq \frac{t^{\alpha-2}(1-t)^{2}}{\alpha-1}\left(r-\mu C_{0}\right)>0
$$

so for $t \in[0,1]$ we have

$$
\begin{aligned}
(A y)(t) & =\mu \int_{0}^{1} G(t, s) a(s) f^{*}(s, y(s)-\phi(s)) d s \\
& \leq \mu \int_{0}^{1} q(s) a(s)[g(y(s)-\phi(s))+h(y(s)-\phi(s))] d s \\
& =\mu \int_{0}^{1} q(s) a(s) g(y(s)-\phi(s))\left\{1+\frac{h(y(s)-\phi(s))}{g(y(s)-\phi(s))}\right\} d s \\
& \leq \mu \int_{0}^{1} q(s) a(s) g\left(\frac{s^{\alpha-2}(1-s)^{2}}{\alpha-1}\left(r-\mu C_{0}\right)\right)\left\{1+\frac{h(r)}{g(r)}\right\} d s \\
& \leq \mu K_{0} g\left(\frac{r-\mu C_{0}}{\alpha-1}\right)\left\{1+\frac{h(r)}{g(r)}\right\} \int_{0}^{1} q(s) a(s) g\left(s^{\alpha-2}(1-s)^{2}\right) d s \\
& =\mu K_{0} a_{0} g\left(\frac{r-\mu C_{0}}{\alpha-1}\right)\left\{1+\frac{h\{r\}}{g\{r\}}\right\} .
\end{aligned}
$$

This together with (5.12) yields

$$
\|A y\|=\|A y\|_{[0,1]} \leq r=\|y\|,
$$

so (5.21) is satisfied.

Next we show

$$
\|A y\| \geq\|y\| \text { for } K \cap \partial \Omega_{2} .
$$


To see this let $y \in K \cap \partial \Omega_{2}$ so $\|y\|=\|y\|_{[0,1]}=R$ and $y(t) \geq\left(t^{\alpha-2}(1-t)^{2} /(\alpha-1)\right) R$ for $t \in[0,1]$. Also for $t \in[0,1]$ we have

$$
\begin{aligned}
y(t)-\phi(t)=y(t)-\mu w(t) & \geq \frac{t^{\alpha-2}(1-t)^{2}}{\alpha-1} R-\mu C_{0} \frac{t^{\alpha-2}(1-t)^{2}}{\alpha-1} \\
& \geq \frac{t^{\alpha-2}(1-t)^{2}}{\alpha-1} R\left(1-\frac{\mu C_{0}}{R}\right) \geq \frac{\epsilon t^{\alpha-2}(1-t)^{2}}{\alpha-1} R .
\end{aligned}
$$

As a result

$$
y(t)-\phi(t) \geq \frac{\epsilon \theta^{\alpha}}{\alpha-1} R \quad \text { for } t \in[\theta, 1-\theta]
$$

We have

$$
\begin{aligned}
(A y)(\sigma) & =\mu \int_{0}^{1} G(\sigma, s) q(s) f^{*}(s, y(s)-\phi(s)) d s \\
& \geq \mu \int_{0}^{1-\theta} G(\sigma, s) q(s)\left[g_{1}(y(s)-\phi(s))+h_{1}(y(s)-\phi(s))\right] d s \\
& =\mu \int_{0}^{1-\theta} G(\sigma, s) q(s) g_{1}(y(s)-\phi(s))\left\{1+\frac{h_{1}(y(s)-\phi(s))}{g_{1}(y(s)-\phi(s))}\right\} d s \\
& \geq \mu g_{1}(R) \int_{0}^{1-\theta} G(\sigma, s) q(s)\left\{1+\frac{h_{1}\left(\left(\epsilon \theta^{\alpha} /(\alpha-1)\right) R\right)}{g_{1}\left(\left(\epsilon \theta^{\alpha} /(\alpha-1)\right) R\right)}\right\} d s .
\end{aligned}
$$

This together with (5.14) yields

$$
(A y)(\sigma) \geq R=\|y\|
$$

Thus $\|A y\| \geq\|y\|$, so (5.25) is held.

Now Theorem 2.5 implies that $A$ has a fixed-point $y \in K \cap\left(\bar{\Omega}_{2} \backslash \Omega_{1}\right)$, that is, $r \leq$ $\|y\|=\|y\|_{[0.1]} \leq R$ and $y(t) \geq t^{\alpha-2}(1-t)^{2} r$ for $t \in[0,1]$. Thus $y(t)$ is a solution of (5.16) with $y(t)>\phi(t)$ for $t \in(0,1)$. Thus (5.1) has a positive solution $u(t)=y(t)-\phi(t)>$ for $t \in(0,1)$.

Example 5.3. Consider the boundary value problem

$$
\begin{gathered}
\mathbf{D}_{0+}^{\alpha} y(t)=\mu\left(y^{-a}(t)+y^{b}(t)-1\right), \quad 0<t<1,3<\alpha \leq 4, \\
y(0)=y(1)=y^{\prime}(0)=y^{\prime}(1)=0, \quad 0<a<1<b,
\end{gathered}
$$

where $\mu \in\left(0, \mu_{0}\right)$ is such that

$$
\frac{(\alpha-1)^{2}(\alpha-2) \mu_{0}}{2 \Gamma(\alpha)}+(\alpha-1)\left(2 \mu_{0} a_{0}\right)^{1 / \alpha} \leq 1,
$$


here

$$
a_{0}=\int_{0}^{1} q(s) a(s) g\left(s^{\alpha-2}(1-s)^{2}\right) d s=\int_{0}^{1} s^{1-a(\alpha-1)}(1-s)^{(\alpha-1)} d s<\infty .
$$

Then (5.30) has a solution $y$ with $y(t)>0$ for $t \in(0,1)$.

To see this we will apply Theorem 5.2 with (here $R>1$ will be chosen later, in fact here we choose $R>1$ so that $\epsilon=1 / 2$ works, i.e., we choose $R$ so that $1-(\mu /(2 \Gamma(\alpha) R)) \geq 1 / 2)$ ),

$$
\begin{gathered}
g(y)=g_{1}(y)=y^{-a}, \quad h(y)=h_{1}(y)=y^{b}, \quad a(t)=1, e(t)=t^{-1 / 2}, \\
\mathrm{a} K_{0}=1, \epsilon=\frac{1}{2}, \quad \theta=\frac{1}{4}, \quad C_{0}=\frac{(\alpha-1)^{2}(\alpha-2)}{\Gamma(\alpha)} \int_{0}^{1} s^{-1 / 2} d s=\frac{(\alpha-2)^{2}(\alpha-2)}{2 \Gamma(\alpha)} .
\end{gathered}
$$

Clearly (5.7)-(5.11) and (5.13) hold. Now (5.12) holds with $r=1$ since

$$
\begin{aligned}
\mu K_{0} a_{0}=\mu a_{0}<\mu_{0} a_{0} \leq \frac{1}{2}\left(\frac{1-\mu_{0} C_{0}}{\alpha-1}\right)^{a} & \leq \frac{1}{2}\left(\frac{1-\mu c_{0}}{\alpha-1}\right)^{a} \\
& =\frac{r}{\{1+(h(r) / g(r))\} g\left(\left(r-\mu M C_{0}\right) /(\alpha-1)\right)}
\end{aligned}
$$

from (5.31). Finally notice (5.14) is satisfied for $R$ large since

$$
\frac{R g_{1}\left(\epsilon \theta^{\alpha} /(\alpha-1)\right) R}{g_{1}(R) g_{1}\left(\left(\epsilon \theta^{\alpha} /(\alpha-1)\right) R\right)+g_{1}(R) h_{1}\left(\left(\epsilon \theta^{\alpha} /(\alpha-1)\right) R\right)}=\frac{R^{1+a}}{1+(\alpha-1)^{-(a+b)}\left[\epsilon \theta^{\alpha}\right]^{a+b} R^{a+b}} \longrightarrow 0,
$$

as $R \rightarrow \infty$, since $b>1$. Thus all the conditions of Theorem 5.2 are satisfied so existence is guaranteed.

\section{Acknowledgments}

This paper is supported by Key Subject of Chinese Ministry of Education (no. 109051) and Scientific Research Fund of Heilongjiang Provincial Education Department (no. 11544032).

\section{References}

[1] O. P. Agrawal, "Formulation of Euler-Lagrange equations for fractional variational problems," Journal of Mathematical Analysis and Applications, vol. 272, no. 1, pp. 368-379, 2002.

[2] S. Zhang, "Existence of positive solution for some class of nonlinear fractional differential equations," Journal of Mathematical Analysis and Applications, vol. 278, no. 1, pp. 136-148, 2003.

[3] D. Delbosco and L. Rodino, "Existence and uniqueness for a nonlinear fractional differential equation," Journal of Mathematical Analysis and Applications, vol. 204, no. 2, pp. 609-625, 1996.

[4] I. Podlubny, Fractional Differential Equations, vol. 198 of Mathematics in Science and Engineering, Academic Press, San Diego, Calif, USA, 1999. 
[5] S. G. Samko, A. A. Kilbas, and O. I. Marichev, Fractional Integrals and Derivatives: Theory and Applications, Gordon and Breach, Yverdon, Switzerland, 1993.

[6] M. A. Krasnosel'skiı̌, Positive Solutions of Operator Equations, P. Noordhoff, Groningen, The Netherlands, 1964.

[7] R. W. Leggett and L. R. Williams, "Multiple positive fixed points of nonlinear operators on ordered Banach spaces," Indiana University Mathematics Journal, vol. 28, no. 4, pp. 673-688, 1979.

[8] Z. Bai and H. Lü, "Positive solutions for boundary value problem of nonlinear fractional differential equation," Journal of Mathematical Analysis and Applications, vol. 311, no. 2, pp. 495-505, 2005.

[9] S. Zhang, "The existence of a positive solution for a nonlinear fractional differential equation," Journal of Mathematical Analysis and Applications, vol. 252, no. 2, pp. 804-812, 2000.

[10] Q. Yao, "Positive solutions for Eigenvalue problems of fourth-order elastic beam equations," Applied Mathematics Letters, vol. 17, no. 2, pp. 237-243, 2004.

[11] W. Li and M. Zhang, "Non-degeneracy and uniqueness of periodic solutions for some superlinear beam equations," Applied Mathematics Letters, vol. 22, no. 3, pp. 314-319, 2009.

[12] Q. Yao, "Existence and multiplicity of positive solutions to a singular elastic beam equation rigidly fixed at both ends," Nonlinear Analysis: Theory, Methods \& Applications, vol. 69, no. 8, pp. 2683-2694, 2008.

[13] M. Galewski, "On the nonlinear elastic beam equation," Applied Mathematics and Computation, vol. 202, no. 1, pp. 427-434, 2008. 


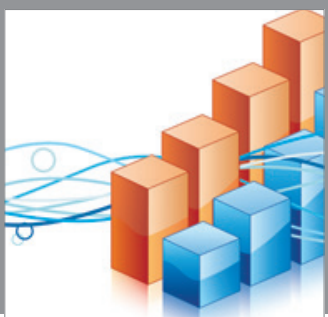

Advances in

Operations Research

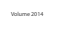

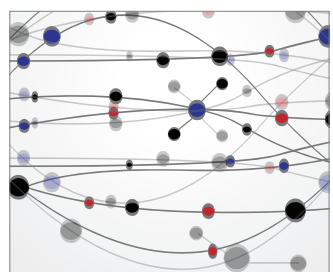

\section{The Scientific} World Journal
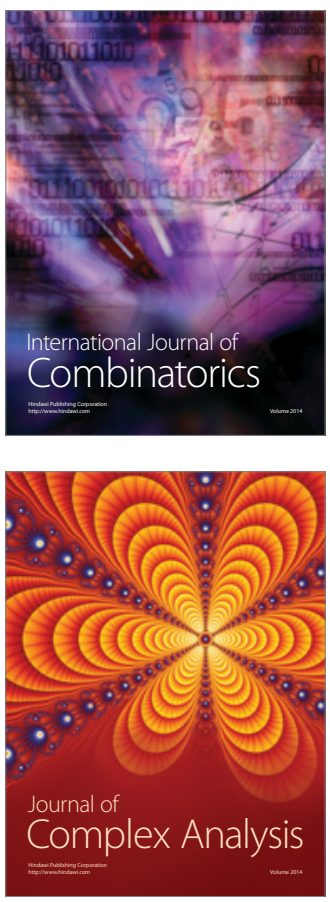

International Journal of

Mathematics and

Mathematical

Sciences
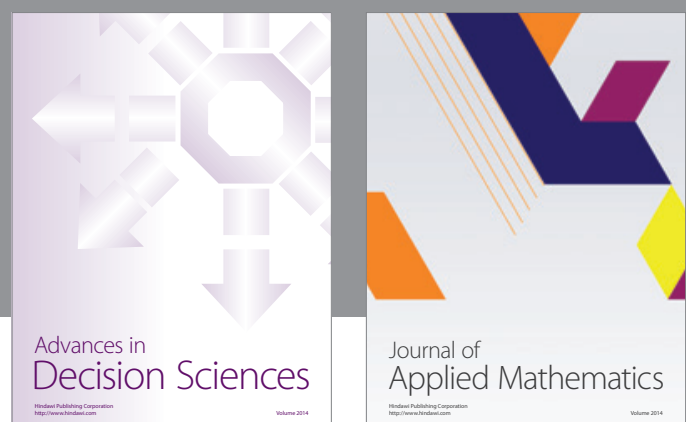

Journal of

Applied Mathematics
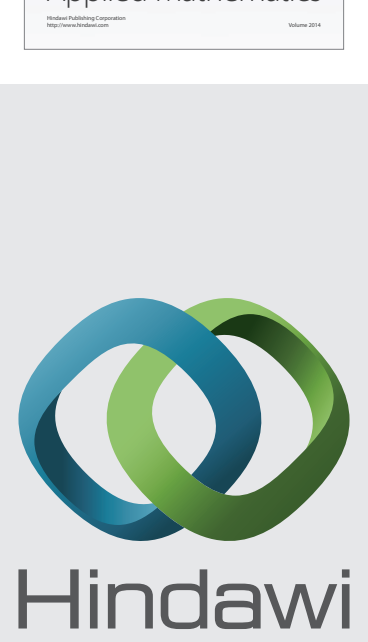

Submit your manuscripts at http://www.hindawi.com
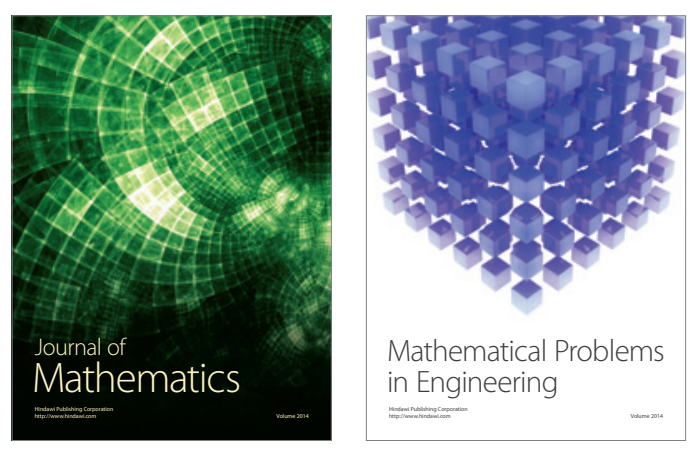

Mathematical Problems in Engineering
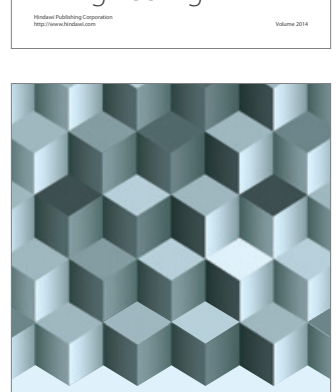

Journal of

Function Spaces
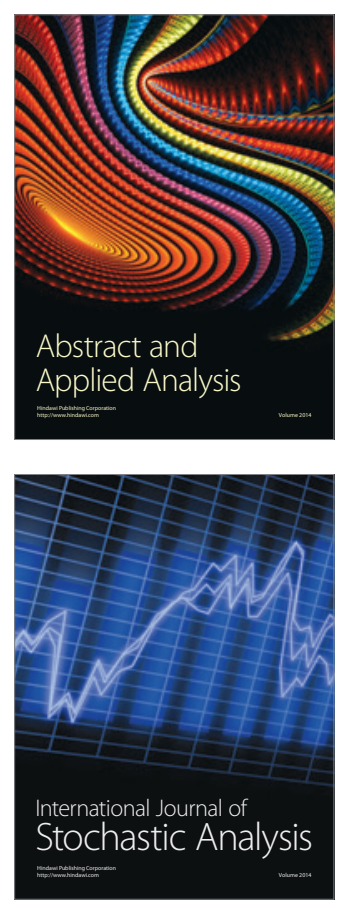

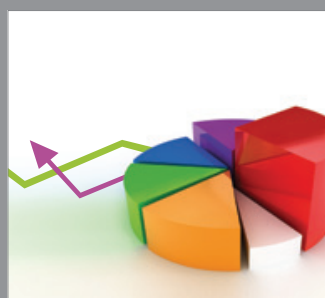

ournal of

Probability and Statistics

Promensencen
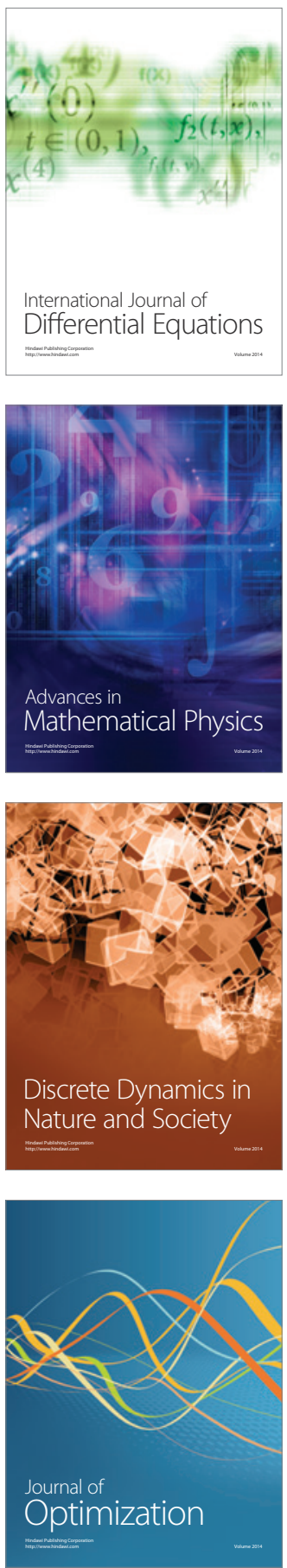